\title{
Effect of pore size of monofilament woven filter cloth as supporting material for dynamic membrane filtration on performance using aerobic membrane bioreactor technology
}

\begin{abstract}
Dynamic membranes (DMs) defined as cake layer forms on a support material such as filter cloth have been of great interest in recent years. The usage of DMs can reduce the capital cost significantly and energy consumption during wastewater treatment by replacing the conventional membrane. The formation of an active dynamic cake layer is highly related to the retention of particles on the support material surface. In this study, different pore sizes of monofilament woven filter cloth made of polypropylene (PP; 20, 40, and $60 \mu \mathrm{m}$ ) were installed as a flat sheet in a DM bioreactor (DMBR) to treat high strength food wastewater $[>1,000 \mathrm{mg} / \mathrm{L}$ chemical oxygen demand (COD)]. The wastewater treatment performances, namely COD, turbidity, total solid separation, and total ammonia nitrogen removal efficiencies, were assessed daily. The system achieved average COD removals higher than $80 \%$ and $70 \%$ for smaller pore size and larger pore size, respectively. Based on the results (duration of 4 days), the $20 \mu \mathrm{m}$ pore size monofilament filter cloth was found most suitable for the cake layer development. The results also demonstrate that the biofilm composed of the cake layer of the DM on smaller pore size significantly concurs with the high treatment efficiency compared with the larger pore size.
\end{abstract}

